\title{
MODELING MULTI-WAVELENGTH STELLAR ASTROMETRY. II. DETERMINING ABSOLUTE INCLINATIONS, GRAVITY-DARKENING COEFFICIENTS, AND SPOT PARAMETERS OF SINGLE STARS WITH SIM LITE
}

\author{
JefFrey L. Coughlin ${ }^{1,3}$, Thomas E. Harrison ${ }^{1}$, And Dawn M. Gelino ${ }^{2}$ \\ ${ }^{1}$ Department of Astronomy, New Mexico State University, P.O. Box 30001, MSC 4500, Las Cruces, NM 88003-8001, USA; jlcough@nmsu.edu \\ ${ }^{2}$ NASA Exoplanet Science Institute, California Institute of Technology, Pasadena, CA 91125, USA \\ Received 2010 May 6; accepted 2010 September 8; published 2010 October 21
}

\begin{abstract}
We present a novel technique to determine the absolute inclination of single stars using multi-wavelength submilliarcsecond astrometry. The technique exploits the effect of gravity darkening, which causes a wavelengthdependent astrometric displacement parallel to a star's projected rotation axis. We find that this effect is clearly detectable using SIM Lite for various giant stars and rapid rotators, and present detailed models for multiple systems using the REFLUX code. We also explore the multi-wavelength astrometric reflex motion induced by spots on single stars. We find that it should be possible to determine spot size, relative temperature, and some positional information for both giant and nearby main-sequence stars utilizing multi-wavelength SIM Lite data. These data will be extremely useful in stellar and exoplanet astrophysics, as well as supporting the primary SIM Lite mission through proper multi-wavelength calibration of the giant star astrometric reference frame, and reduction of noise introduced by starspots when searching for extrasolar planets.
\end{abstract}

Key words: astrometry - stars: fundamental parameters

Online-only material: color figures

\section{INTRODUCTION}

SIM Lite is currently expected to have $\sim 80$ spectral channels (Davidson et al. 2009), spanning 450-900 nm, thus allowing multi-wavelength microarcsecond astrometry, which no current or planned ground- or space-based astrometric project (GAIA, CHARA, VLT/PRIMA, etc.) is able to match. We showed in our first paper (Coughlin et al. 2010), hereafter referred to as Paper I, the implications multi-wavelength microarcsecond astrometry has for interacting binary systems. In this paper, we discuss an interesting effect we encountered while modeling binary systems, namely, that gravity darkening in stars produces a wavelength-dependent astrometric offset from the center of mass that increases with decreasing wavelength. It is possible to use this effect to derive both the inclination and gravitydarkening exponent of a star in certain cases.

Determining the absolute inclination of a given star has many practical applications. There is much interest in the formation of binary stars, where whether or not the spin axis of each star is aligned with the orbital axis provides insight into the formation history of the system (Turner et al. 1995). The mutual inclination between the stellar spin axes and the orbital axis can greatly affect the rate of precession, which is used to probe stellar structure and test general relativity (Sterne 1939a, 1939b, 1939c; Kopal 1959; Jeffery 1984). Albrecht et al. (2009) recently reconciled a 30 year old discrepancy between the observed and predicted precession rate of DI Herculis through observations which showed the stellar spin axes were nearly perpendicular to the orbital axis. Along similar lines, extrasolar planets discovered via the radial velocity technique only yield the planetary mass as a function of the inclination of the orbit (Mayor \& Queloz 1995; Noyes et al. 1997; Marcy \& Butler 2000), and thus, if one assumes the planetary orbit and stellar rotation axes are nearly parallel, determining the absolute

\footnotetext{
3 NSF Graduate Research Fellow.
}

inclination of the host star yields the absolute mass of the planet. If the stellar spin axis is found not to be parallel to the planetary orbital axis, this provides valuable insights into the planet's formation, migration, and tidal evolution histories (Winn et al. 2006; Fabrycky \& Winn 2009). A final example is the study of whether or not the spin axes of stars in clusters are aligned, which both reveal insight into their formation processes as well as significantly affect the determination of the distances to those clusters (Jackson \& Jeffries 2010).

Our proposed technique can also be used in conjunction with other methods of determining stellar inclination to yield more precise inclination values and other stellar parameters of interest. Gizon \& Solanki (2003) and Ballot et al. (2006) have shown that one can derive the inclination of the rotation axis for a given star using the techniques of asteroseismology given high-precision photometry with continuous coverage over a long baseline, such as that provided by the CoRoT and Kepler missions. This technique is sensitive to rotation rates as slow as the Sun's, but becomes easier with faster rotation rates. Domiciano de Souza et al. (2004) discuss how spectro-interferometry can yield both the inclination angle and amount of differential rotation for a star, parameterized by $\alpha$. For both eclipsing binaries and transiting planets, the observation of the Rossiter-McLaughlin (RM) effect can yield the relative co-inclination between the two components (Winn et al. 2006; Albrecht et al. 2009; Fabrycky \& Winn 2009). The technique we propose in this paper would be complementary to these techniques in several ways. First, it would provide an independent check on the derived inclination axis from each method, confirming or refuting the asteroseismic models and spectro-interferometric and RM techniques. Second, in principle the asteroseismic technique is not dependent on the gravity-darkening coefficient $\beta_{1}$, and the spectro-interferometric technique is correlated with the value for $\alpha$; combining techniques would yield direct and robust observationally determined values for $i, \alpha$, and $\beta_{1}$. Finally, the accurate, observational determination of $\alpha$ and $\beta_{1}$ (along with 
stellar limb darkening) is critical to accurately deriving the coinclination from the RM effect, as well as other quantities in stellar and exoplanet astrophysics.

In this paper, we also present models for multiple systems and discuss the determination of spot location, temperature, and size on single stars, which produce a wavelength-dependent astrometric signature as they rotate in and out of view. Starspots are regions on the stellar surface where magnetic flux emerges from bipolar magnetic regions, which blocks convection and thus heat transport, effectively cooling the enclosed gas, and thus are fundamental indicators of stellar magnetic activity and the internal dynamos that drive it. Işik et al. (2007) discuss how the observation of spot location, duration, stability, and temperature can probe the stellar interior and constrain models of magnetic flux transport. Through the observation of the rotation rates of starspots at varying latitudes, one is able to derive the differential rotation rate of the star (Collier Cameron 2002), which may be directly related to the frequency of starspot cycles. Mapping spots in binary star systems provide insight into the interaction between the magnetic fields of the two components, which can cause orbital period changes (Applegate 1992), radii inflation (López-Morales 2007; Morales et al. 2008), and may possibly explain the $\sim 2-3 \mathrm{hr}$ period gap in cataclysmic variable systems (Watson et al. 2007). Detecting and characterizing starspots via multi-wavelength astrometry would be complementary to other existing techniques, namely, optical interferometry (Wittkowski et al. 2002), tomographic imaging (Donati et al. 2006; Aurière et al. 2008), photometric monitoring (Alekseev 2004; Mosser et al. 2009), and in the future, microlensing (Hwang \& Han 2010).

We present the details of our modeling code, REFLUX, in Section 2, discuss the inclination effect and present models for multiple stars in Section 3, discuss the spot effects and present models in Section 4, and present our conclusions in Section 5.

\section{THE REFLUX CODE}

REFLUX ${ }^{4}$ is a code that computes the flux-weighted astrometric reflex motion of binary systems. We discussed the code in detail in Paper I, but in short, it utilizes the Eclipsing Light Curve (ELC) code, which was written to compute light curves of eclipsing binary systems (Orosz \& Hauschildt 2000). The ELC code represents the surfaces of two stars as a grid of individual luminosity points, and calculates the resulting light curve given the provided systemic parameters. ELC includes the dominant physical effects that shape a binary's light curve, such as non-spherical geometry due to rotation, gravity darkening, limb darkening, mutual heating, reflection effects, and the inclusion of hot or cool spots on the stellar surface. For the work in this paper we have simply turned off one of the stars, thus allowing us to probe the astrometric effects of a single star. To compute intensity, ELC can either use a blackbody formula or interpolate from a large grid of NextGen model atmospheres (Hauschildt et al. 1999). For all the simulations in this paper, we have used the model atmosphere option, and will note now, and discuss in more detail later, that the calculation of limb darkening is automatically included in NextGen model atmospheres. These artificially derived limb-darkening coefficients have recently been shown to be in error by as much as $\sim 10 \%-20 \%$ in comparison to observationally derived values (Claret 2008), and thus their

\footnotetext{
$4 \quad$ REFLUX can be run via a web interface from

http://astronomy.nmsu.edu/jlcough/reflux.html. Additional details as to how to
} set up a model are presented there. uncertainties must be included, although for this work, due to symmetry, we find the introduced error is negligible. For all our simulations, we model the $U, B, V, R, I, J, H$, and $K$ bands for completeness and comparison to future studies, though we note that SIM Lite will not be able to observe in the $U, J, H$, or $K$ bandpasses.

\section{INCLINATION AND ROTATION}

The astrophysical phenomenon of gravity darkening, also sometimes referred to as gravity brightening, is the driving force behind the ability to determine the inclination of a single star using multi-wavelength astrometry. A rotating star is geometrically distorted into an oblate spheroid, such that its equatorial radius is greater than its polar radius, and thus the poles have a higher surface gravity, and the equator a lower surface gravity, than a non-rotating star with the same mass and average radius. This increased surface gravity, $g$, at the poles results in a higher effective temperature, $T_{\text {eff }}$, and thus luminosity; decreased $g$ at the equator results in a lower $T_{\text {eff }}$ and luminosity. This temperature and luminosity differential cause the star's center of light, or photocenter, to be shifted toward the visible pole, away from the star's gravitational center of mass. Since the inclination determines how much of the pole is visible, the amount of displacement between the photocenter and the center of mass is directly related to the inclination. Furthermore, since the luminosity difference effectively results from a ratio of blackbody luminosities of differing temperatures, the effect is wavelength dependent, with shorter wavelengths shifted more than longer wavelengths. Thus, the amount of displacement between the measured photocenter in two or more wavelengths is directly related to the inclination. See Figure 1 for an illustration of the effect.

An additional complicating factor is the exact dependence of temperature on local gravity. von Zeipel (1924) was the first to derive the quantitative relationship between them, showing that $T_{\mathrm{eff}}^{4} \propto g^{\beta_{1}}$, where $\beta_{1}$ is referred to as the gravity-darkening exponent. The value of $\beta_{1}$ has been a subject of much study and debate; for a complete review, see Claret (2000), who presents both an excellent discussion of past studies, as well as new, detailed computations of $\beta_{1}$ using modern models of stellar atmospheres and internal structure that encompass stars from 0.08 to $40 M_{\odot}$. Since the value of $\beta_{1}$ affects the temperature differential between equator and pole, the multi-wavelength displacement will also be dependent on the value of $\beta_{1}$. The total amplitude of the effect will be scaled by the angular size of the star, which depends on both its effective radius and distance. Thus, in total, the components of this inclination effect are the effective stellar radius, distance, effective temperature, rotation rate, $\beta_{1}$, and inclination of the star. In principle, one is able to determine the effective stellar radius, effective temperature, rotation rate, and distance of a target star using groundbased spectroscopy and space-based parallax measurements, including from SIM Lite. Thus, when modeling the multiwavelength displacement of the stellar photocenter, the only two components that need to be solved for are the inclination and $\beta_{1}$, with $\beta_{1}$ already having some constraints from theory.

A good trio of stars for modeling and testing this inclination effect is the components of the binary system Capella (Aa and $\mathrm{Ab}$ ) and the single star Vega. Torres et al. (2009) have very recently published an extremely detailed analysis of both the binary orbit of Capella and the physical and evolutionary states of the individual components, providing both new observations, as well as drawing from the previous observations and analyses 

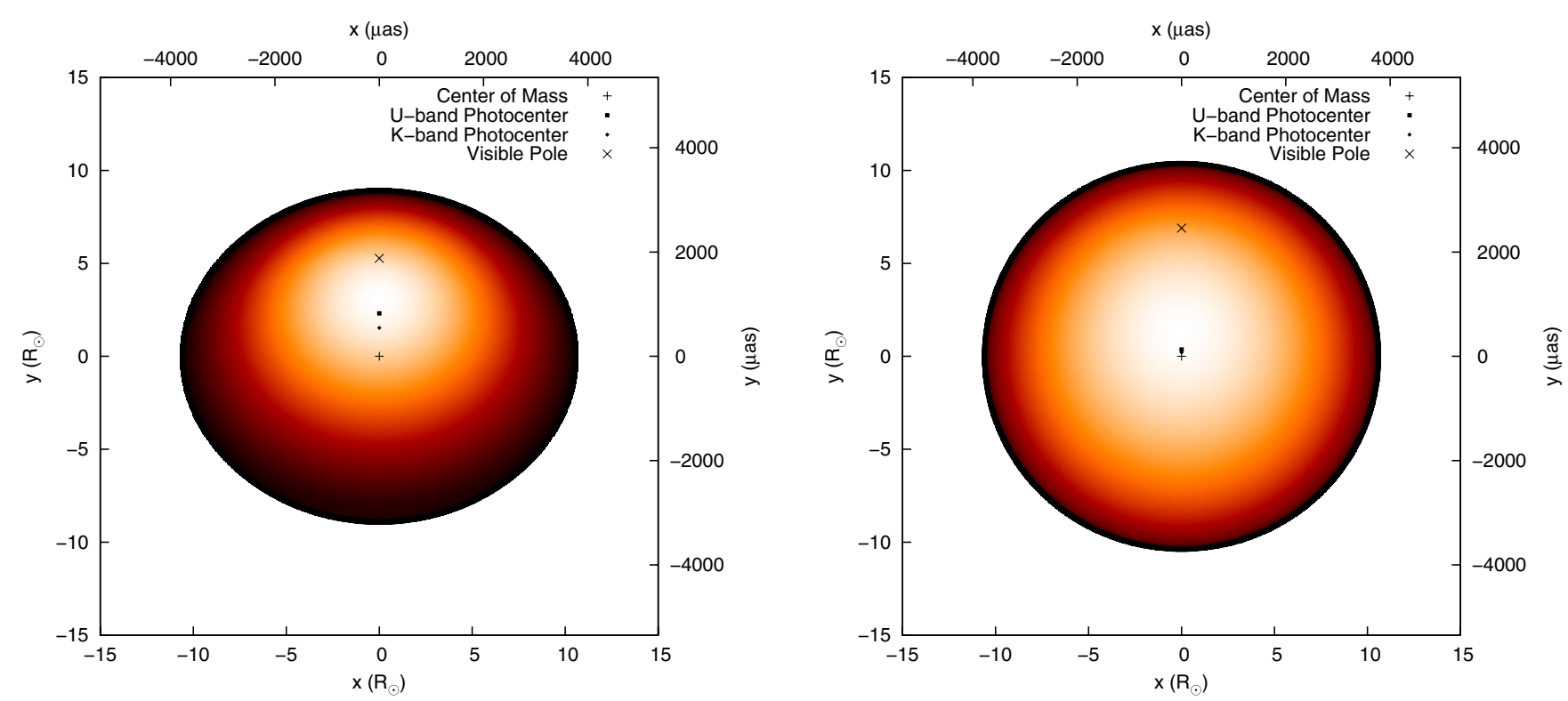

Figure 1. Illustration of the inclination effect using brightness maps of Capella Ab, which has an inclination of 42.788 (Torres et al. 2009). Left: Capella Ab artificially spun-up to near its break-up speed to accentuate the gravity-darkening effect for ease of viewing. As can be seen, the photocenter of the system is dramatically shifted away from the center of mass, toward the visible pole, which is brighter than the rest of the star due to gravity darkening. Furthermore, since the pole is physically hotter, the $U$-band photocenter is shifted more than the $K$-band photocenter, and in the direction of the projected rotation axis or the $y$-axis. Right: Capella Ab at its actual rotation period. As can be seen, the actual effect is small compared to the angular size of the star on the sky, but still large compared to the $1 \mu$ as benchmark of SIM Lite. The presence of limb darkening is clearly visible, which causes a decrement in flux toward the limb of the star. Note that the broad wavelength coverage of $S I M$ Lite will only cover the $B, V, R$, and $I$ bandpasses.

(A color version of this figure is available in the online journal.)

Table 1

Parameters for Capella Aa

\begin{tabular}{lc}
\hline \hline \multicolumn{1}{c}{ Parameter } & Value $^{\mathrm{a}}$ \\
\hline Distance (pc) & 12.9 \\
Rotation period (days) & 106.0 \\
Mass $\left(M_{\odot}\right)$ & 2.70 \\
Radius $\left(R_{\odot}\right)$ & 12.2 \\
Effective temperature $(\mathrm{K})$ & 4940 \\
$\beta_{1}$ & 0.43 \\
\hline
\end{tabular}

Note. ${ }^{\text {a }}$ Values from Torres et al. (2009).

of Hummel et al. (1994) and Strassmeier et al. (2001). Vega, in addition to being one of the most well-studied stars in the sky, has recently been discovered to be a very rapid rotator seen nearly pole-on (Aufdenberg et al. 2006; Peterson et al. 2006; Hill et al. 2010). In total, these three stars represent both slow and rapid rotators for giant and main-sequence stars at a range of temperatures, as Capella $\mathrm{Aa}$ is a slow-rotating K-type giant, Capella $\mathrm{Ab}$ is a fast-rotating G-type giant, and Vega is a very fast rotating A-type main-sequence star. With many ground-based interferometric observations to compare with, and being bright and nearby, these stars also present excellent targets for SIM Lite.

We use the REFLUX code to generate models of the astrometric displacement from $U$ band to $H$ band, with respect to the $K$-band photocenter, for inclinations from $0^{\circ}$ to $90^{\circ}$, for each star, as shown in Figures 2-4. We use systemic parameters given by Torres et al. (2009) for Capella $\mathrm{Aa}$ and $\mathrm{Ab}$, and by Aufdenberg et al. (2006) and Peterson et al. (2006) for Vega, listed in Tables 1, 2, and 3, respectively. We employ the model atmospheres incorporated into the ELC code, as well as automatically chosen values for $\beta_{1}$ based on Figure 1 of Claret (2000). Additionally, in each figure we show a dashed
Table 2

Parameters for Capella $\mathrm{Ab}$

\begin{tabular}{lc}
\hline \hline \multicolumn{1}{c}{ Parameter } & Value $^{\mathrm{a}}$ \\
\hline Distance $(\mathrm{pc})$ & 12.9 \\
Rotation period (days) & 8.64 \\
Mass $\left(M_{\odot}\right)$ & 2.56 \\
Radius $\left(R_{\odot}\right)$ & 9.2 \\
Effective temperature $(\mathrm{K})$ & 5700 \\
$\beta_{1}$ & 0.39 \\
\hline
\end{tabular}

Note. ${ }^{a}$ Values from Torres et al. (2009).

Table 3

Parameters for Vega

\begin{tabular}{lc}
\hline \hline \multicolumn{1}{c}{ Parameter } & Value $^{\mathrm{a}}$ \\
\hline Distance (pc) & 7.76 \\
Rotation period (days) & 0.521 \\
Mass $\left(M_{\odot}\right)$ & 2.11 \\
Radius $\left(R_{\odot}\right)$ & 2.5 \\
Effective temperature $(\mathrm{K})$ & 9602 \\
$\beta_{1}$ & 1.02 \\
\hline
\end{tabular}

Note.

${ }^{a}$ Values from Aufdenberg et al. (2006) and Peterson et al. (2006).

line to indicate the effect of decreasing the gravity-darkening coefficient by $10 \%$ to simulate the uncertainty of the models (Claret 2000) and explore the correlation with other parameters.

As can be seen from these models, we find that the effect is quite large for a Capella Ab-like or Vega-like fast rotator, but only marginally detectable for a slower rotating system like Capella Aa. This also implies that this effect would not be detectable for a slow-rotating, main-sequence star like our Sun. 


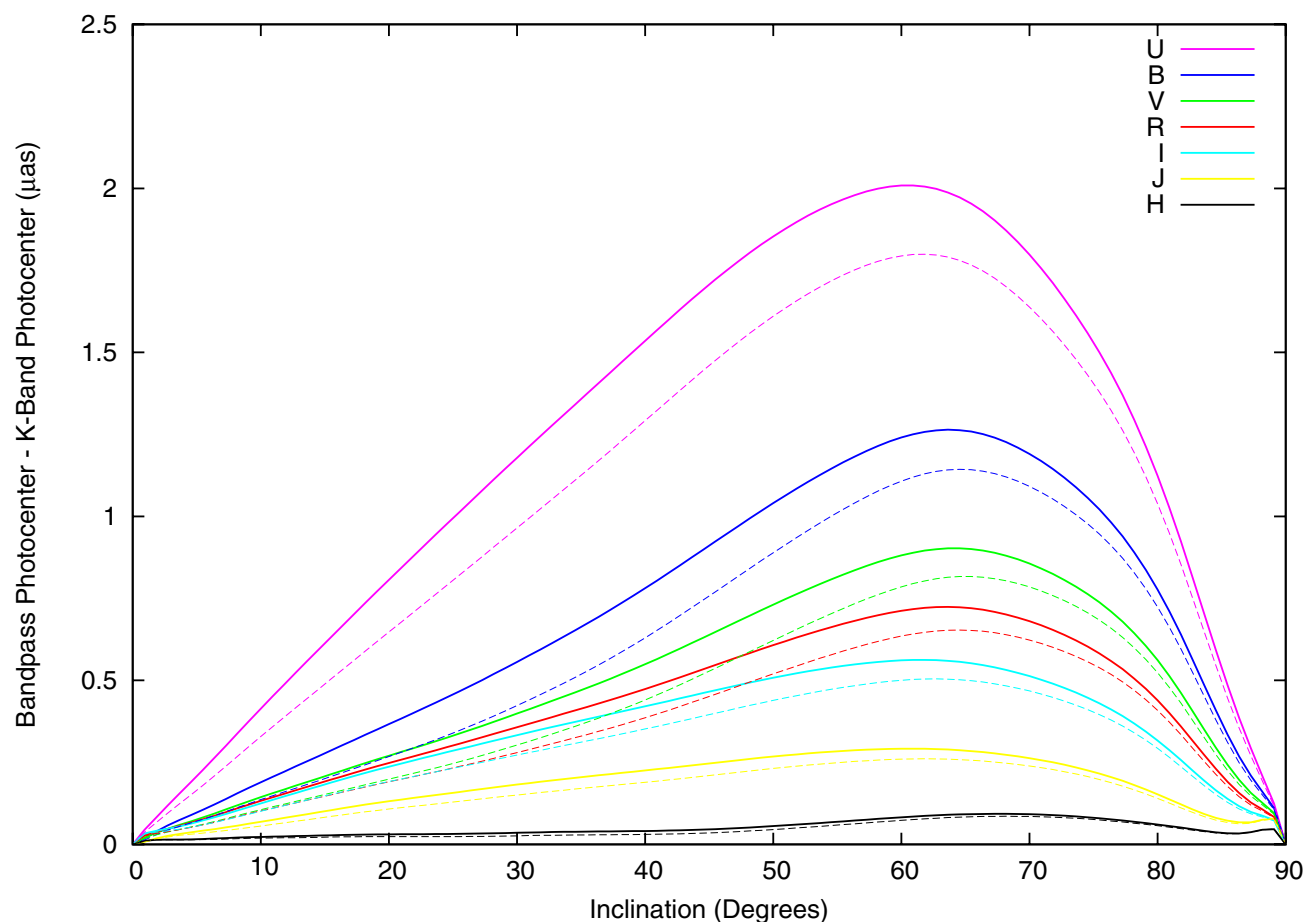

Figure 2. Astrometric displacement of each bandpass with respect to $K$ band vs. inclination for Capella Aa. Dashed lines are a model with $\beta_{1}$ decreased by $10 \%$. Due to the slow rotation rate of Capella Aa, the effect is limited to a maximum of $\sim 2.0 \mu$ as between $U$ and $K$ bands, and only a maximum of $\sim 0.7 \mu$ as between $B$ and $I$ bands, where SIM Lite will operate. This puts the detection of this effect for Capella Aa at the very edge of SIM Lite's capability.

(A color version of this figure is available in the online journal.)

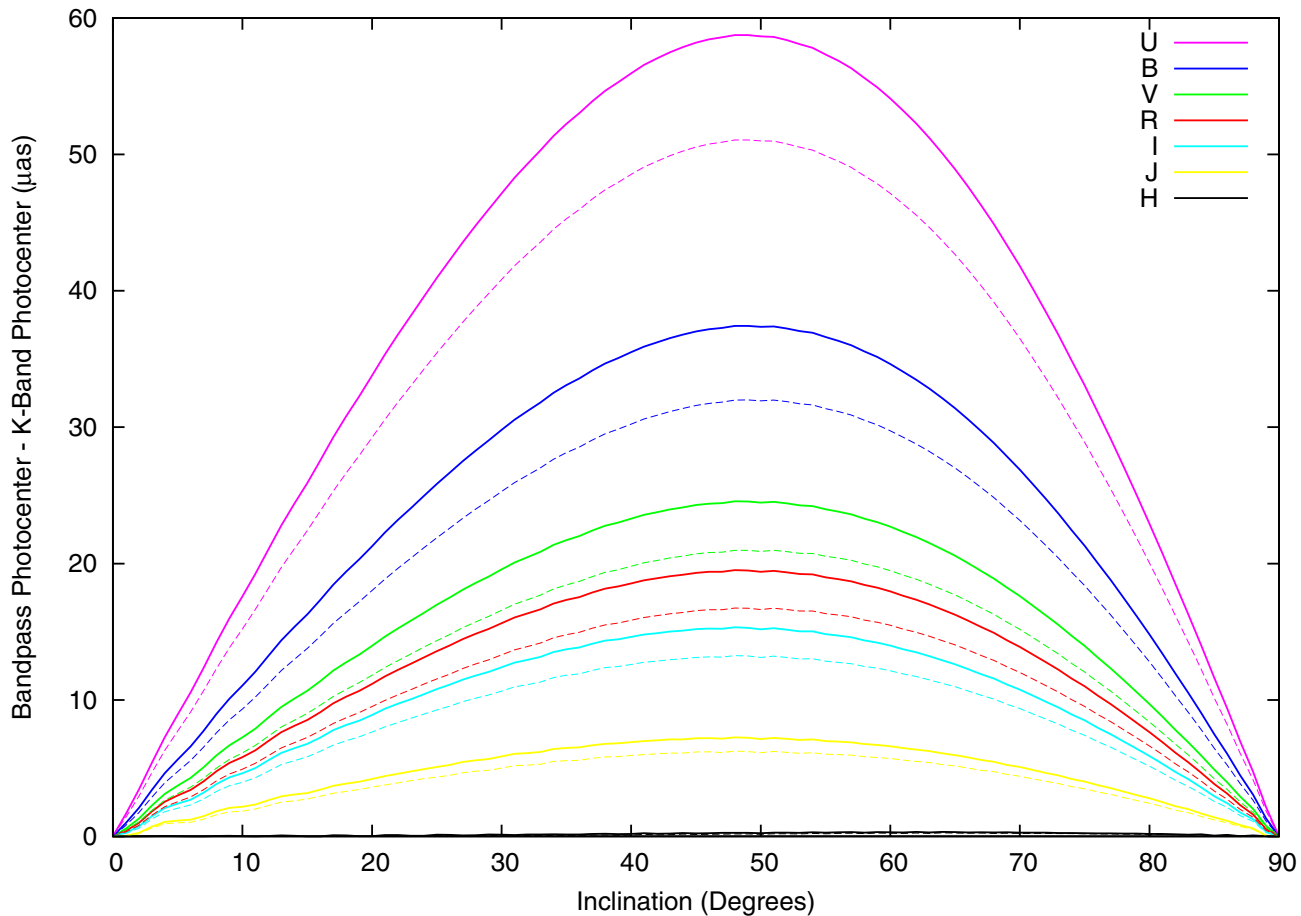

Figure 3. Astrometric displacement of each bandpass with respect to $K$ band vs. inclination for Capella Ab. Dashed lines are a model with $\beta_{1}$ decreased by $10 \%$. Due to the fast rotation rate of a Capella Ab like star, the effect is moderate with tens of microarcseconds of displacement, and thus these types of stars are excellent targets for SIM Lite. The actual inclination of Capella Ab is 42.788 (Torres et al. 2009), and thus Capella Ab itself should show a large shift of the photocenter with wavelength. Note that the broad wavelength coverage of $S I M$ Lite will only cover the $B, V, R$, and $I$ bandpasses.

(A color version of this figure is available in the online journal.)

Our modeling confirms this, showing a total U-K amplitude of $\ll 0.1 \mu$ as for a $1.0 M_{\odot}, 1.0 R_{\odot}$ star with a rotation period of 30.0 days at 10.0 parsec. These conclusions on detectability are made with the assumption that, for bright stars like these, SIM Lite can achieve its microarcsecond benchmark. We show that this is possible in a narrow angle (NA) mode by employing the 


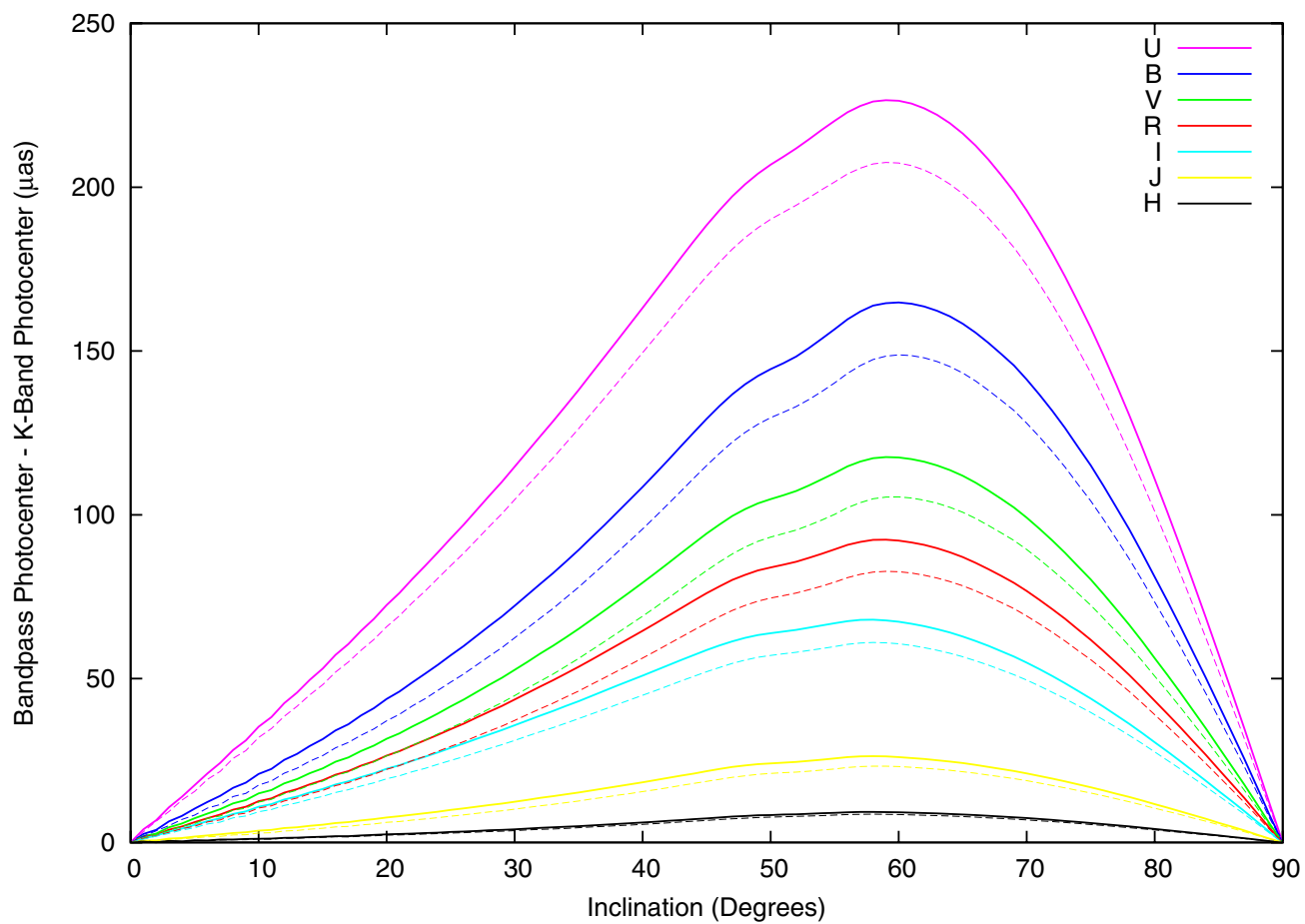

Figure 4. Astrometric displacement of each bandpass with respect to $K$ band vs. inclination for Vega. Dashed lines are a model with $\beta_{1}$ decreased by $10 \%$. Note that due to the very fast rotation of Vega, along with a high value of $\beta_{1}$, the effect can be quite large, at a couple of hundred microarcseconds. For a Vega-like star, SIM Lite observations would yield very accurate values for $\beta_{1}$ and the inclination. For Vega itself, which is known to be nearly pole on, with an inclination of 5.7 (Hill et al. 2010), there should be a $B$-band minus $I$-band displacement of $6.0 \mu$ as, still detectable by SIM Lite. Note that the broad wavelength coverage of SIM Lite will only cover the $B, V, R$, and $I$ bandpasses.

(A color version of this figure is available in the online journal.)

SIM Differential Astrometry Performance Estimator (DAPE; Plummer 2009). For a target star with magnitude $V=5$, and a single comparison star with $V=10$ located within a degree of it on the sky, by integrating $15 \mathrm{~s}$ on the target, and $30 \mathrm{~s}$ on the reference, for 10 visits at five chop cycles each, a final precision of $\pm 1.01 \mu$ as is achieved in only $1.04 \mathrm{hr}$ of the total mission time. For a fainter target with $V=10$, this precision is only reduced to $\pm 1.32 \mu$ as in the same amount of mission time. In utilizing NA mode, one must be careful in choosing the reference star(s) to ensure that they are not stars with a substantial wavelengthdependent centroid. Given the only constraints on reference stars are that they need to have $V \lesssim 10$ and are within $1^{\circ}$ on the sky, one could easily choose a slow-rotating, main-sequence star, determined as such via ground-based observations as a wavelength-independent astrometric reference star. We also note that wide angle SIM Lite measurements, with a precision of $\sim 5 \mu$ as, may not detect the wavelength-dependent photocenter of a system like Capella, but will have no difficulty detecting it in stars like Capella $\mathrm{Ab}$ or Vega.

The effect of decreasing the gravity-darkening exponent is to decrease the total amplitude of the effect in each wavelength, with shorter wavelengths affected more than longer wavelengths. Thus, the choice of gravity-darkening exponent is intimately tied to the derived inclination. If one were to model observed data with a gravity-darkening exponent that was $\sim 10 \%$ different than the true value, one would derive an inclination that would also be $\sim 10 \%$ different from the true inclination. However, the two combinations of inclination and gravity-darkening exponent do not produce identical results, and can be distinguished with a sufficient precision at a number of wavelengths. For example, if one were to adopt the nominal value for $\beta_{1}$ and derive an inclination of $40^{\circ}$ for a Vega-like star, then adopt a $\beta_{1}$ value that was $10 \%$ lower, one would derive an inclination of $43^{\circ}$, a $7.5 \%$ change. In this case though, with the lower $\beta_{1}$ value, the measured photocenter in the $U, B, V, R, I, J$, and $H$ bandpasses, with respect to the $K$-band photocenter, would differ from the nominal $\beta_{1}$ model by $\sim 0.5,-1.0,-2.0,-2.0$, $-1.6,-1.0$, and $0.2 \mu$ as, respectively. Note that for $B, V, R$, and $I$, where SIM Lite can observe, these discrepancies, on the order of $\sim 1.0 \mu$ as, should be large enough to be distinguished in the NA mode. Thus, a unique solution exists for the values of $i$ and $\beta_{1}$ if the photocenter is measured in three or more wavelengths. (The photocenter of one wavelength is used as a base measurement, with respect to which the photocenters of other wavelengths are measured. If one measures the photocenter in three or more wavelengths, one then has two or more differential photocenter measurements, and can then solve for the two unknown variables. Although we have chosen the $K$ band as the base measurement in our models, simply because it is the longest wavelength we model and thus shows the least variation, one could easily choose another band, such as $R$, where SIM Lite will be able to observe. In the example just given of possible degeneracy between inclination and $\beta_{1}$, if one chose the $R$ band as the base measurement, one would still detect a difference of $\sim 1.0 \mu$ as between the $B$ and $V$ photocenters, and be able to resolve the degeneracy between inclination and $\beta_{1}$.)

Another complication is the possibility of having equally good fitting high and low solutions for $i$. For example, if one observed and determined a best-fit inclination of $70^{\circ}$ for a Vegalike star, one could obtain a reasonably good fit as well at $46^{\circ}$, (see Figure 4). However, just as in the case of the uncertainty in the value of $\beta_{1}$, discernible discrepancies would exist. In this case, the discrepancies in the measured photocenter in the $U$, $B, V, R, I, J$, and $H$ bandpasses, with respect to the $K$-band 
photocenter, would be $\sim 0.1,-9.0,-2.0,1.5,6.0,1.0$, and $0.2 \mu \mathrm{as}$, respectively. Just as in the case of the uncertainty in the value of $\beta_{1}$, this discrepancy between equally good fitting highand low-inclination solutions can be resolved if one has three or more wavelengths obtained in the NA mode.

As mentioned in Section 1, we note that the limb-darkening function, which was automatically chosen by the ELC code as incorporated into the model atmospheres, can differ from actual observed values by $\sim 10 \%$ (Claret 2008). We have tested how changing the limb-darkening coefficients by $10 \%$ affects the resulting astrometric displacements, and find that the result is less than $0.5 \%$ for all wavelengths, and thus is negligible in the modeling. The reason is that limb darkening is symmetric, and thus while increased limb darkening damps the visible pole, it also damps the rest of the star, and thus the relative brightness between regions is maintained.

Additionally, this inclination technique yields the orientation of the projected stellar rotation axis on the sky, which is parallel to the wavelength dispersion direction. When coupled with the derived inclination, this technique thus yields the full threedimensional orientation of the rotation axis. This could be a powerful tool in determining the overall alignment of stellar axes in the local neighborhood and in nearby clusters.

\section{STARSPOTS}

Another area of astrophysical interest to which multiwavelength astrometric measurements from SIM Lite can contribute is the study of starspots. As the cause of starspots are intense magnetic fields at the photosphere, they are typically found in stars with convective envelopes, especially rapidly rotating stars. Thus, both low-mass, main-sequence $\mathrm{K}$ and $\mathrm{M}$ dwarfs, as well as rapidly rotating giant and sub-giant stars, are known to host large spots on their surface. The study of the distribution, relative temperature, and size of these spots would greatly contribute to the study of magnetic field generation in stellar envelopes. A starspot that rotates in and out of view will cause a shift of the photocenter for a single star, which has been a subject of much recent discussion in the literature (e.g., Hatzes 2002; Unwin 2005; Eriksson \& Lindegren 2007; Catanzarite et al. 2008; Makarov et al. 2009; Lanza et al. 2008), especially in light of its potential to mimic, or introduce noise when characterizing, an extrasolar planet. However, there has been no mention in the literature of the multi-wavelength astrometric signature of stellar spots, where, just as in the case of the gravity-darkening inclination effect, we are looking at essentially two blackbodies with varying temperatures, and thus shorter wavelengths will be more affected by a spot than longer wavelengths.

To characterize the multi-wavelength astrometric signature of stellar spots, we model two spotty systems, again using the REFLUX code. We model Capella $\mathrm{Ab}$, which shows evidence of large spots and is suspected of being an RS CVn variable (Hummel et al. 1994), and a typical main-sequence K dwarf. For Capella Ab, we use the parameters listed in Table 2, along with the star's determined inclination of 42.788 (Torres et al. 2009), and add a cool spot that has a temperature that is $60 \%$ of the average surface temperature, located at the equator, at a longitude such that it is seen directly at phase $270^{\circ}$, and having an angular size of $10^{\circ}$ (where $90^{\circ}$ would cover exactly one half of the star). For the K dwarf system, we use the physical parameters listed in Table 4, simulating a typical K Dwarf at $10 \mathrm{pc}$, and add a cool spot with the same parameters as we do for Capella Ab. Additionally, to investigate the effects of cool versus hot spots
Table 4

Parameters for the K Dwarf System

\begin{tabular}{lc}
\hline \hline \multicolumn{1}{c}{ Parameter } & Value \\
\hline Distance $(\mathrm{pc})$ & 10.0 \\
Inclination $\left(^{\circ}\right)$ & 60.0 \\
Period $($ days $)$ & 20 \\
Mass $\left(M_{\odot}\right)$ & 0.6 \\
Radius $\left(R_{\odot}\right)$ & 0.6 \\
Effective temperature $(\mathrm{K})$ & 4500 \\
Latitude of spot $\left(^{\circ}\right)$ & 90 \\
Longitude of spot $\left(^{\circ}\right)$ & 270 \\
Angular Size of Spot $\left(^{\circ}\right)$ & 10 \\
Cool spot temperature factor & 0.6 \\
Hot spot temperature factor & 1.4 \\
\hline
\end{tabular}

or flares, we also run a model with a hot spot by changing the spot temperature to be $40 \%$ greater than the average surface temperature. We present our models in Figures 5-7.

As can be seen for Capella Ab, the gravity-darkening inclination effect presented in Section 3 dominates the spread of colors in the $y$-direction, the direction parallel to the stars' projected rotation axis. However, the amplitude of the spot motion is quite large, with a total amplitude of $\sim 40 \mu$ as in all bandpasses, which would be easily detectable by SIM Lite. For the K dwarf with a cool spot, we see a much smaller, but still detectable shift of amplitude $\sim 5-8 \mu$ as, depending on the wavelength. In the case of a hot spot or flare, we see a much larger displacement, on the order of $\sim 10-200 \mu \mathrm{as}$, depending on the wavelength, which would be easily detectable by SIM and provide extremely precise values in deriving the spot parameters.

In general, the temperature of the spot, in relation to the mean stellar surface temperature, is related to the spread in observed wavelengths, with a larger spread indicating a larger temperature difference. The duration of the astrometric displacement in phase, coupled with the overall amplitude of the astrometric displacement, yields the size of the spot, as larger spots will cause larger displacements and be visible for a larger amount of rotational phase. The latitude of the spot can also affect the total duration. Finally, the amplitude of the astrometric displacement in the $x$ direction versus the $y$ direction is dependent on both the latitude of spot and the inclination of the star. Thus, when modeled together, one is able to recover these parameters. This work can also be combined with our work in Paper I to derive the location of spots in binary systems, as the astrometric signature of the spot is simply added to the astrometric signature of the binary system.

The astrometric motion induced upon a parent star by a host planet does not have a wavelength dependence. Spots however, as we have shown via our modeling, have a clear wavelength dependence. Thus, if one has a candidate planetary signal from astrometry, but it shows a wavelength-dependent motion, it must then be a false positive introduced from starspots at the rotation period of the star (assuming that the planet's emitted flux is negligible compared to the star). Furthermore, when SIM is launched, there will likely be many cases where a marginally detectable signal due to a planetary companion is found at a very different period than the rotation period of the star. However, starspots will still introduce extra astrometric jitter which will degrade the signal from the planetary companion. Multi-wavelength astrometric data can be used to model and remove the spots, which will have a wavelength dependence, and thus strengthen the planetary signal, which will not have a wavelength dependence. 

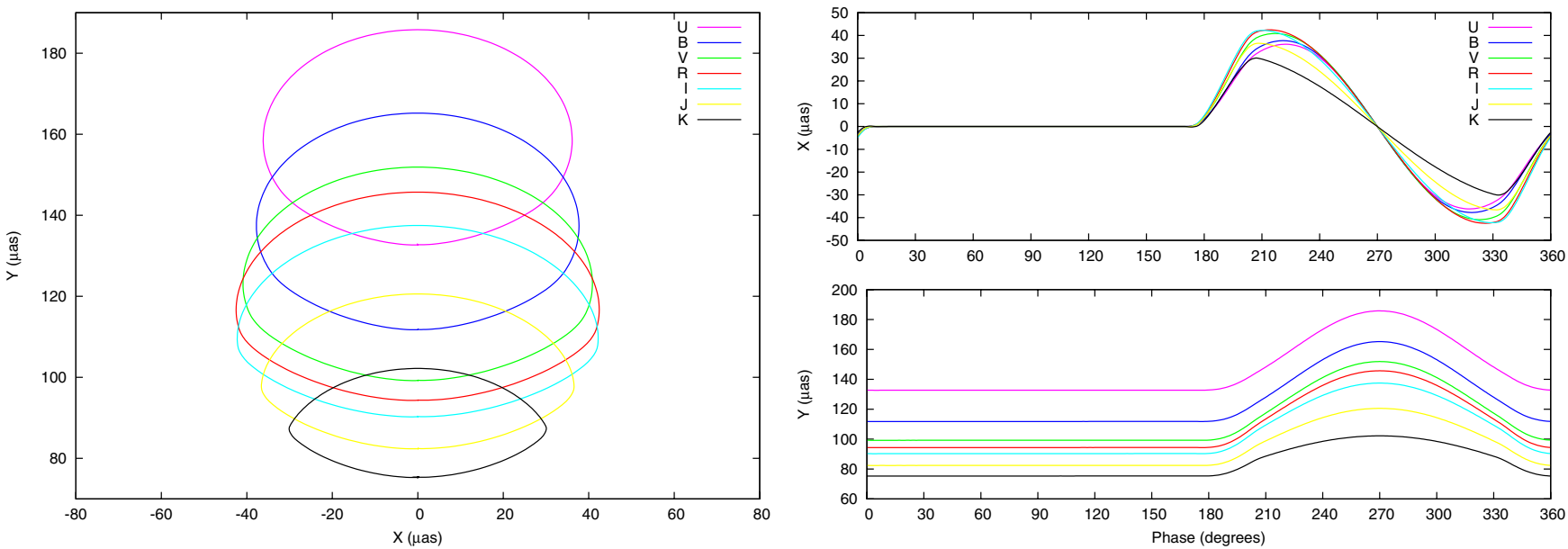

Figure 5. Simulated cool spot on Capella Ab. The spot is located on the equator, with a longitude such that it is seen directly at phase $270^{\circ}$. The strong presence of the gravity-darkening effect, discussed in Section 3, dominates the wavelength spread in the $y$ direction. Note that the broad wavelength coverage of SIM Lite will only cover the $B, V, R$, and $I$ bandpasses.

(A color version of this figure is available in the online journal.)
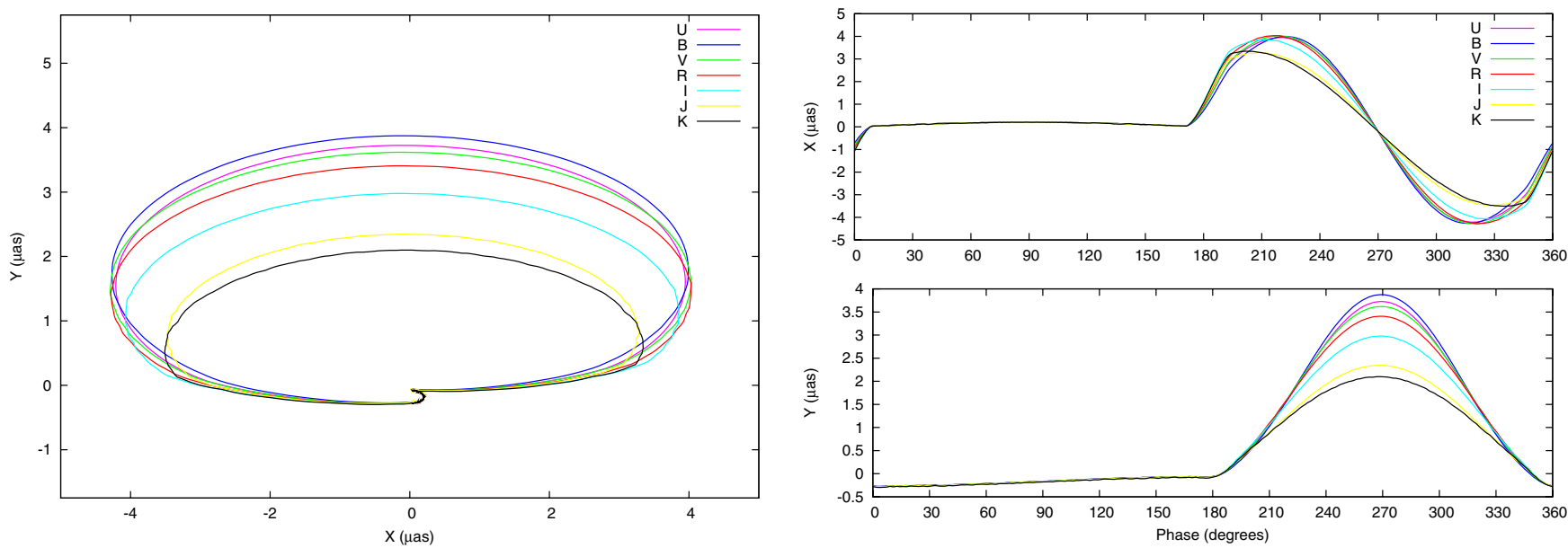

Figure 6. Simulated cool spot on a nearby K dwarf star with an inclination of $60^{\circ}$, whose parameters are given in Table 4 . The spot is located on the equator, with a longitude such that it is seen directly at phase $270^{\circ}$. Note that the broad wavelength coverage of SIM Lite will only cover the $B, V, R$, and $I$ bandpasses.

(A color version of this figure is available in the online journal.)
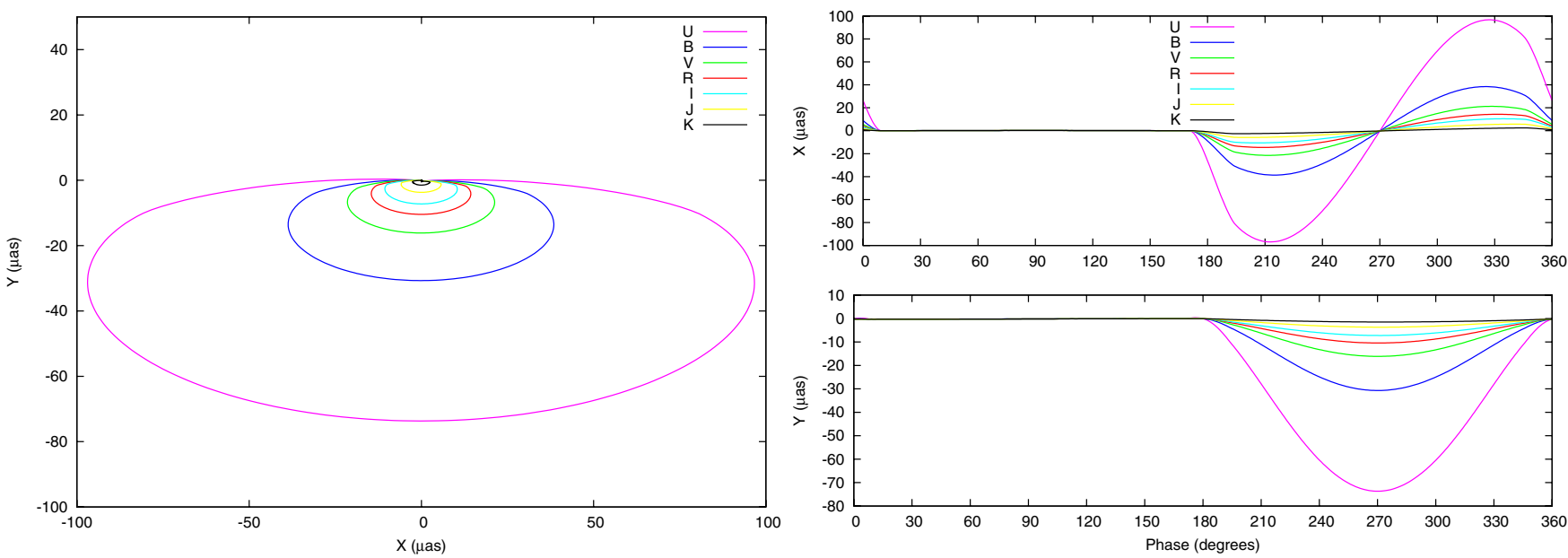

Figure 7. Simulated hot spot or flare on a nearby K dwarf star with an inclination of $60^{\circ}$, whose parameters are given in Table 4 . The spot is located on the equator, with a longitude such that it is seen directly at phase $270^{\circ}$. Note that the broad wavelength coverage of $S I M$ Lite will only cover the $B, V, R$, and $I$ bandpasses.

(A color version of this figure is available in the online journal.) 


\section{DISCUSSION AND CONCLUSION}

We have presented detailed models of the multi-wavelength astrometric displacement that SIM Lite will observe due to gravity darkening and stellar spots using the REFLUX code. We find that SIM Lite observations, especially when combined with other techniques, will be able to determine the absolute inclination, gravity-darkening exponent, and three-dimensional orientation of the rotational axis for fast- and slow-rotating giant stars and fast-rotating main-sequence stars. This technique will be especially useful in probing binary star and exoplanet formation and evolution, as well as the physics of star-forming regions. Direct observational determination of the gravitydarkening exponent has direct applications in both stellar and exoplanet astrophysics. This technique is also relatively inexpensive in terms of SIM Lite observing time, as one need only to observe a given star once, as opposed to binary stars and planets, which require constant monitoring over an entire orbit. It should be noted that this effect should be taken into account when constructing the SIM Lite astrometric reference frame, such that fast-rotating giants should be excluded so as not to produce a wavelength-dependent astrometric reference frame.

We have also presented models of starspots on single stars and find that SIM Lite should be able to discern their location, temperature, and size. Combined with other techniques, this will provide great insight into stellar differential rotation, magnetic cycles and underlying dynamos, and magnetic interaction in close binaries. From this modeling, it should especially be noted that multi-wavelength astrometry is a key tool in the hunt for extrasolar planets, either by ruling out false signals created by spots or simply removing extra astrometric jitter introduced by spots. Thus, it remains critical that SIM Lite maintains a multiwavelength astrometric capability in its final design.

This work was sponsored in part by a SIM Science Study (PI: D. Gelino) from the National Aeronautics and Space Administration through a contract with the Jet Propulsion Laboratory, California Institute of Technology. J.L.C. acknowledges additional support from a New Mexico Space Grant Consortium Fellowship. We thank the referee for comments which greatly helped to improve this manuscript, particularly in making the presentation of our ideas much clearer.

\section{REFERENCES}

Albrecht, S., Reffert, S., Snellen, I. A. G., \& Winn, J. N. 2009, Nature, 461, 373 Alekseev, I. Y. 2004, Sol. Phys., 224, 187

Applegate, J. H. 1992, ApJ, 385, 621
Aufdenberg, J. P., et al. 2006, ApJ, 645, 664

Aurière, M., et al. 2008, A\&A, 491, 499

Ballot, J., García, R. A., \& Lambert, P. 2006, MNRAS, 369, 1281

Catanzarite, J., Law, N., \& Shao, M. 2008, Proc. SPIE, 7013, 70132K

Claret, A. 2000, A\&A, 359, 289

Claret, A. 2008, A\&A, 482, 259

Collier Cameron, A. 2002, Astron. Nachr., 323, 336

Coughlin, J. L., et al. 2010, ApJ, 717, 776

Davidson, J., Edberg, S., Danner, R., Nemati, B., \& Unwin, S. (ed.) 2009, SIM Lite: Astrometric Observatory (Pasadena, CA: NASA JPL)

Domiciano de Souza, A., Zorec, J., Jankov, S., Vakili, F., Abe, L., \& JanotPacheco, E. 2004, A\&A, 418, 781

Donati, J., Forveille, T., Cameron, A. C., Barnes, J. R., Delfosse, X., Jardine, M. M., \& Valenti, J. A. 2006, Science, 311, 633

Eriksson, U., \& Lindegren, L. 2007, A\&A, 476, 1389

Fabrycky, D. C., \& Winn, J. N. 2009, ApJ, 696, 1230

Gizon, L., \& Solanki, S. K. 2003, ApJ, 589, 1009

Hatzes, A. P. 2002, Astron. Nachr., 323, 392

Hauschildt, P. H., Allard, F., \& Baron, E. 1999, ApJ, 512, 377

Hill, G., Gulliver, A. F., \& Adelman, S. J. 2010, ApJ, 712, 250

Hummel, C. A., Armstrong, J. T., Quirrenbach, A., Buscher, D. F., Mozurkewich, D., Elias, II, N. M., \& Wilson, R. E. 1994, AJ, 107, 1859

Hwang, K., \& Han, C. 2010, ApJ, 709, 327

Issik, E., Schüssler, M., \& Solanki, S. K. 2007, A\&A, 464, 1049

Jackson, R. J., \& Jeffries, R. D. 2010, MNRAS, 402, 1380

Jeffery, C. S. 1984, MNRAS, 207, 323

Kopal, Z. 1959, in Close Binary Systems, ed. Z. Kopal (London: Chapman \& Hall)

Lanza, A. F., De Martino, C., \& Rodonò, M. 2008, New Astron., 13, 77

López-Morales, M. 2007, ApJ, 660, 732

Makarov, V. V., Beichman, C. A., Catanzarite, J. H., Fischer, D. A., Lebreton, J., Malbet, F., \& Shao, M. 2009, ApJ, 707, L73

Marcy, G. W., \& Butler, R. P. 2000, PASP, 112, 137

Mayor, M., \& Queloz, D. 1995, Nature, 378, 355

Morales, J. C., Ribas, I., \& Jordi, C. 2008, A\&A, 478, 507

Mosser, B., Baudin, F., Lanza, A. F., Hulot, J. C., Catala, C., Baglin, A., \& Auvergne, M. 2009, A\&A, 506, 245

Noyes, R. W., Jha, S., Korzennik, S. G., Krockenberger, M., Nisenson, P., Brown, T. M., Kennelly, E. J., \& Horner, S. D. 1997, ApJ, 483, L111

Orosz, J. A., \& Hauschildt, P. H. 2000, A\&A, 364, 265

Peterson, D. M., et al. 2006, Nature, 440, 896

Plummer, K. 2009, TaPE Webtool User Guide 1.1

Sterne, T. E. 1939a, MNRAS, 99, 451

Sterne, T. E. 1939b, MNRAS, 99, 662

Sterne, T. E. 1939c, MNRAS, 99, 670

Strassmeier, K. G., Reegen, P., \& Granzer, T. 2001, Astron. Nachr., 322, 115

Torres, G., Claret, A., \& Young, P. A. 2009, ApJ, 700, 1349

Turner, J. A., Chapman, S. J., Bhattal, A. S., Disney, M. J., Pongracic, H., \& Whitworth, A. P. 1995, MNRAS, 277, 705

Unwin, S. C. 2005, in ASP Conf. Ser. 338, Astrometry in the Age of the Next Generation of Large Telescopes, ed. P. K. Seidelmann \& A. K. B. Monet (San Francisco, CA: ASP), 37

von Zeipel, H. 1924, MNRAS, 84, 665

Watson, C. A., Steeghs, D., Shahbaz, T., \& Dhillon, V. S. 2007, MNRAS, 382, 1105

Winn, J. N., et al. 2006, ApJ, 653, L69

Wittkowski, M., Schöller, M., Hubrig, S., Posselt, B., \& von der Lühe, O. 2002, Astron. Nachr., 323, 241 